\title{
Evaluation of 19-gauge endoscopic ultrasonography aspiration needles using various echoendoscopes
}

Authors

Institutions
Takao Itoi ${ }^{1}$, Fumihide Itokawa' ${ }^{1}$, Atsushi Sofuni' ${ }^{1}$ Toshio Kurihara' ${ }^{1}$, Takayoshi Tsuchiya' ${ }^{1}$, Kentaro Ishii', Shujiro Tsuji', Nobuhito Ikeuchi' ${ }^{1}$, Hiroshi Kawakami², Fuminori Moriyasu' ${ }^{1}$, Ichiro Yasuda ${ }^{3}$

${ }^{1}$ Department of Gastroenterology and Hepatology, Tokyo Medical University, Tokyo, Japan

${ }^{2}$ Department of Gastroenterology, Hokkaido University Graduate School of Medicine, Sapporo, Japan

${ }^{3}$ First Department of Internal Medicine, Gifu University Hospital, Gifu

\section{Bibliography}

Dol http://dx.doi.org/

10.1055/s-0033-1359212

Endoscopy International Open

2013; 1: E24-E30

(c) Georg Thieme Verlag KG

Stuttgart · New York

E-ISSN 2196-9736

Corresponding author:

Takao Itoi, M.D., FASGE

Department of

Gastroenterology and

Hepatology, Tokyo Medical

University

6-7-1 Nishishinjuku

Shinjuku-ku, Tokyo 160-0023

Japan

itoi@tokyo-med.ac.jp
Background and study aims: 19-gauge (19G) needles are used for EUS-guided tissue acquisition and interventions. The aim of the current study was to compare the functional characteristics of 19G EUS needles by means of using various echoendoscopes in a bench simulator.

Methods: The angles achieved for 19G needles (EchoTip: ET-19G, EchoTip Flex: ExF-19G, Expect: Ex-19G, and ProCore: PC-19G) and for the distal tip of the echoendoscope were evaluated for maximal angulation settings of the distal tip and the elevator. Also the resistance to advancement of the $19 \mathrm{G}$ needles at these endoscope settings was assessed. All evaluations were done with endoscopes in a straight and in a curved position.

Results: There was no large discrepancy for scope and needle angles among all echoendoscopes except for the slim Pentax scope (EG-3270UK). The ExF-19G and PC-19G needles showed better optimal angles in various conditions compared to standard 19G needles. In straight scope position,

\section{Introduction}

$\nabla$

Several 19-gauge needles are currently available for endoscopic ultrasonography-guided fine-needle aspiration (EUS-FNA) and interventions [118]. However, the large bore and inherent stiffness of these needles hinder their maneuverability for puncture. In addition, other factors such as scope position (bent or curved), echoendoscope tip angulation, and elevator use affect the maneuverability of the needle.

In a prior study from our group that evaluated one particular 19G FNA needle (EchoTip, Cook Endoscopy, Winston-Salem, N.C.), we found that the needle was suboptimal for performing transduodenal FNA of pancreatic head and uncinate lesions [19]. We observed that when the tip of the echoendoscope was angulated or when the elevator was used, there was increased resistance to passage of the $19 \mathrm{G}$ needle that precluded sam- out of the 20 conditions ( 5 endoscopes $\times 2$ positions of the distal tip $\times 2$ elevator positions) the numbers of optimal angulations achieved for the Ex-19G, ExF-19G, ET-19G, and PC-19G, were 11/ 20 (55\%), 20/20 (100\%), 14/20 (70\%) and 18/20 (90\%), respectively. However, regarding resistance, it was impossible to advance theneedle with $14 / 20$ settings (70\%) for the Ex-19G, 3/20 (15\%) for the ExF-19G, $10 / 20$ (50\%) for the ET19G and $7 / 20$ (35\%) for the PC-19G. When the scopes were bent, with regard to the force needed to advance the needle, the numbers of optimal settings, for the Ex-19G, ExF-19G, ET-19G, and PC-19G, were 1 (5\%), 13 (65\%), 6 (30\%) and 8 (40 $\%)$, respectively. The mean maximum resistance to advancement was less for the ExF-19G than for the other needless in almost all scope and angle conditions $(\mathrm{p}<0.05)$.

Conclusion: Although there was no difference between needles, the resistance to passage was least with the flexible 19-gauge needle (ExF-19G).

pling of lesions. Recently, several 19-gauge needles and small diameter echoendoscopes have been developed. In this study, we compared the characteristics of different 19-gauge EUS needles using various echoendoscopes in a bench simulator (see below) to provide standardized, reproducible, comparative performance data.

\section{Materials and methods}

\section{Echoendoscopes}

Five curved linear array echoendoscopes (GFUCT260, GF-UCT240, and GF-UC240P: Olympus Medical Systems, Tokyo, Japan; EG-3870UTK and EG-3270UK: Pentax, Tokyo, Japan), were examined using the bench simulator. The specifications of these echoendoscopes are described in - Table 1. 


\begin{tabular}{|c|c|c|c|c|}
\hline & \multicolumn{2}{|l|}{ Olympus } & \multicolumn{2}{|l|}{ Pentax } \\
\hline & $\begin{array}{l}\text { GF-UCT260 / } \\
\text { GF-UCT240 }\end{array}$ & GF-UC240P & EG-3870UTK & EG-3270UK \\
\hline \multicolumn{5}{|l|}{ Outer diameter (mm) } \\
\hline Distal end & 14.6 & 14.2 & 14.3 & 12 \\
\hline Insertion tube & 12.6 & 11.8 & 12.8 & 10.8 \\
\hline \multicolumn{5}{|l|}{ Bending section (degree) } \\
\hline Up/down & $130 / 90$ & $130 / 90$ & $130 / 130$ & $130 / 130$ \\
\hline Right/left & $90 / 90$ & $90 / 90$ & $120 / 120$ & $120 / 120$ \\
\hline Working length (mm) & 1,250 & 1,250 & 1,250 & 1,250 \\
\hline Working channel diameter $(\mathrm{mm})$ & 3.7 & 2.8 & 3.8 & 2.8 \\
\hline Elevator angle (degree) & 90 & 90 & 90 & 90 \\
\hline
\end{tabular}

Table 1 Specifications of echoendoscopes.

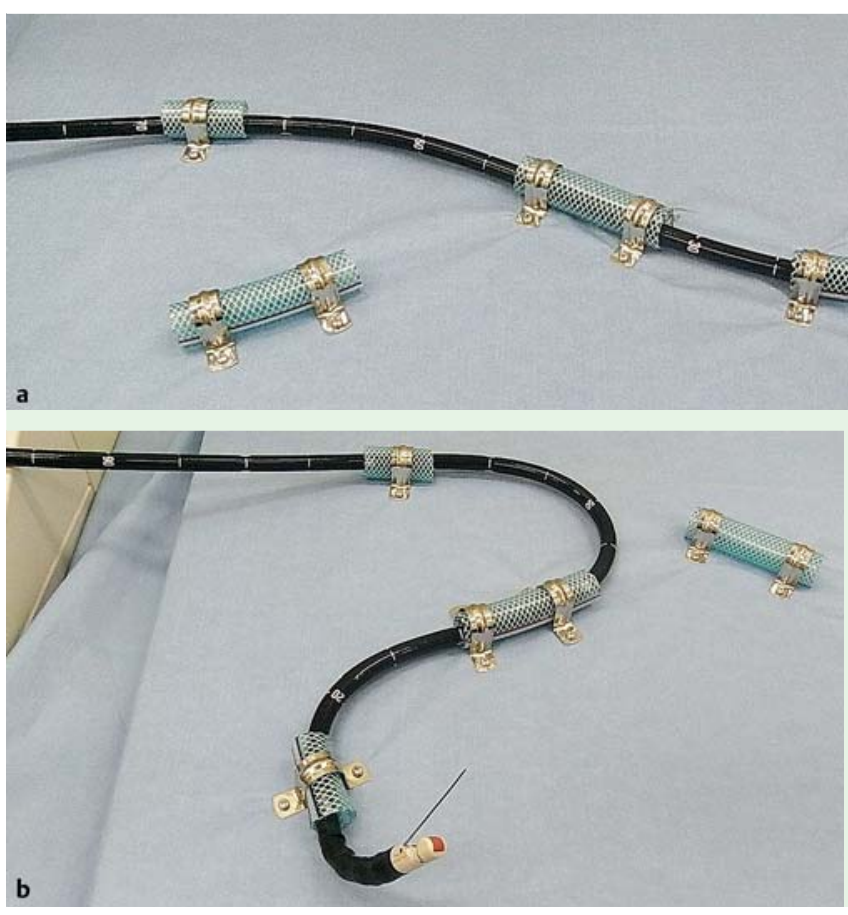

19G needles

The characteristics of commercially available 19-gauge EUS needles were evaluated by bench simulation for both extent of possible angulation and for resistance to passage under various conditions. Four 19-gauge EUS needles were evaluated: 1) Expect ${ }^{\mathrm{TM}}$ standard type (Ex-19G) (Boston Scientific Japan, Tokyo, Japan), 2) Expect ${ }^{\mathrm{TM}}$ flexible type (ExF-19G) (Boston Scientific Japan), 3) EchoTip ${ }^{\circledR}$ ULTRA (ET-19G) (Cook Endoscopy), 4) EchoTip ${ }^{\circledR}$ ProCor$\mathrm{e}^{\mathrm{TM}}$ (PC-19G) (Cook Endoscopy).

Before the bench simulation, the diameter of each needle was accurately measured by a digital micrometer caliper (MDC-25, Mitsutoyo, Kanagawa, Japan).

\section{Bench simulation}

The bench simulator was designed and constructed by one of the authors (T.I.), specifically for the comparative evaluation of different EUS-FNA needles as previously described [19]. The operating portion of the echoendoscope was fixed using a vise, and the insertion portion was fixed in straight $\bullet$ Fig 1 a and curved $\bullet$ Fig $\mathbf{1 b}$ positions using a wooden board, vinyl chloride tubing with a diameter of $19 \mathrm{~mm}$ (ST-19, Toyocs Co., Ltd., Toyama, Japan), and stainless steel saddle grips.

We repeated all the tests with the endoscopes held in both straight and curved positions.
Fig. 1 Bench simulator: a straight therapeutic scope position; $\mathbf{b}$ curved scope position.
Range of angulation of echoendoscope distal tips and consequent angulation of needles

We measured the maximum achievable angulation for each possible combination of the five echoendoscopes and the four 19G needles. The maximum distal tip "up" and elevator "up" settings were used. Thus the "big knob" (up-down) and not the small knob (left-right) of the echoendoscope was used. This was set at $0^{\circ}$ and at maximum in each echoendoscope and the elevator was set at $0^{\circ}$ and $90^{\circ}$ (maximum). When the tip of the needle was flexed, the angle between the echoendoscope and the sheath of the needle was measured.

The echoendoscope angle and the needle angle, were defined as angle A and angle B, respectively, as shown in $\odot$ Fig2. Optimal angulation was defined as angle $A$ and angle $B$ being more than $90^{\circ}$ and $110^{\circ}$, respectively.

All measurements were repeated with the scopes held in both the "straight" and the "curved" positions (see $\bullet$ Fig 1 ).

\section{Resistance of needles}

The resistance to needle advancement through the endoscope was measured using an Autograph (AGS-H, Shimadzu Corporation, Kyoto, Japan), which has a highest tolerable force of 1,000 newtons $(1,000 \mathrm{~N})(\boldsymbol{F i g} 3)$. The needles were advanced at a speed of $500 \mathrm{~mm} / \mathrm{min}$. The length of the needle insertion 
was $30 \mathrm{~mm}$. The resistance to advancement of the needle was recorded continuously during insertion. The data were analyzed by dedicated software (Factory Shikibu 2000, Shimazu Factory Manufacture).

In this study, the resistance of each FNA needle was measured more than 3 times to obtain reproducible results (mean 3.2 times, range 3 to 5 times) using each scope at each of the possible settings. We prepared and used five each of the four 19G needles for the examination.

As there were five scopes, each with two settings of the distal tip (up/down) and two settings of the elevator (up/down), 20 values were obtained for each needle. Again, all of these measurements were repeated with the scopes held in both the "straight" and the "curved" positions (see $\bullet$ Fig 1 ).

We used our prior data for resistance to needle advancement $\left(R_{\text {needle }}\right)$ [18] for grading the resistance: $R_{\text {needle }}<8 \mathrm{~N}$, optimal setting; $R_{\text {needle }}>8 \mathrm{~N}$, suboptimal; $R_{\text {needle }}>11 \mathrm{~N}$, impossible to advance the needle.

\section{Statistics}

Statistical analyses were performed with StatMate III (ATMS Co. Ltd., Tokyo, Japan). Normally distributed data are presented as the mean \pm standard deviation (SD). Kruskal-Wallis test as nonparametric statistic was used to compare between each needle resistances. A P value less than 0.05 was regarded as indicating a statistically significant difference.

\section{Results \\ $\nabla$}

Actually achieved distal tip and needle angulations

Type of echoendoscopes

Olympus The results for all the combinations of scopes and needles, with the scope held in a straight position, are shown in $\bullet$ Table 2. In the straight scope position, for both angle A and angle B, the new therapeutic Olympus scope (GF-UCT260) showed optimal angulation using various 19-gauge needles ( $\bullet$ Table 2 ). However, both angle A and angle B in two echoendoscopes (GFUCT240 and GF-UCP240) using standard FNA needles (Ex-19G and ET-19G) were suboptimal. In the curved scope position, angle $A$ and angle $B$ were each optimal in only one of the 16 possible settings. In both the straight and the curved scope position, the GF-UCT260 allowed in $50 \%$ of possible settings better optimal angles compared to others ( $\bullet$ Table 3 ).

Pentax Regardless of scope positions, the therapeutic Pentax scope (EG-3870UTK) showed optimal angles compared to the slim echoendoscope (E6-3270UK) ( Table3).

\section{The type of needles}

Standard FNA needles (Ex-19G and ET-19G) In the straight scope position, using the Ex-19G showed optimal angles A and B in only two echoendoscopes the (GF-UCT260 and the EG3870 UTK). With the curved scope position, in almost all conditions, angles $\mathrm{A}$ and $\mathrm{B}$ were suboptimal angles. The results for the ET-19G were similar to those of the EX-19G.

PC-19G In the straight scope position, the PC-19G showed optimal angle $A$ and $B$ in all but one echoendoscope (EG-3270UK). Similarly, with the curved scope position, angle B was optimal in all but one echoendoscope (EG-3270UK) though angle $A$ in three echoendoscopes (GF-UCT240, GF-UCP240 and EG-3270UK) was suboptimal.
ExF-19G When the scope was straight, the ExF-19G showed optimal Angle A and B in all but one echoendoscope (EG-3270UK). With the curved scope position, 5 out of the 10 values forof angles A and B were optimal.

Resistance to advancement of 19-gauge needles The maximum resistance to advancement of the FNA needles is shown in $\square$ Table 4 for the straight scope position and in Table 5 for the curved scope position. The results show high reproducibility with only small SDs ( Table 4 and $\bullet$ Table 5 ). In all cases, the resistance to the passage of the FNA needles, $R_{\text {needle, }}$ was greater with greater angulation of the distal tip and of the elevator. Increased resistance was observed irrespective of the type of echoendoscope.

\section{Straight scope position}

With a straight scope, the numbers of optimal settings of the distal tip and the elevator in the Ex-19G, ExF-19G, ET-19G, and PC$19 \mathrm{G}$, were $11 / 20$ (55\%), 20/20 (100\%), 14/20 (70\%) and 18/20 (90\%), respectively ( Table 4$)$. These were the settings where the resistance to advancement of the needle was $\leq 8 \mathrm{~N}$. The mean maximum $R_{\text {needle }}$ was smaller for the ExF-19G than for others in all scope and angle conditions $(\mathrm{p}<0.05)$.

\section{Curved scope position}

With the scope in a curved position, needle advancement was graded as "impossible" in 14/20 settings (70\%) for the Ex-19G, $3 / 20$ (15\%) for the ExF-19G, 10/20 (50\%) for the ET-19G and 7/ 20 (35\%) for the PC-19G ( Table 5). On the other hand, the numbers of optimal settings for the Ex-19G, ExF-19G, ET-19G, and PC19G, were 1 (5\%), 13 (65\%), 6 (30\%) and 8 (40\%), respectively. In almost all conditions, the mean maximum $R_{\text {needle }}$ for the ExF-19G was smaller than that of others $(\mathrm{p}<0.05)$.

\section{Discussion}

$\nabla$

Interventional EUS procedures frequently require the use of a 19gauge needle in conjunction with a 0.035 -inch guidewire. However, most 19-gauge needles, given their large caliber, encounter resistance when the echoendoscope tip is angulated resulting in poor maneuverability. Our experimental study [19] revealed that the distal tipup and elevator-up positions lead to higher resistance for the needle, resulting in difficulty in puncture by the needle and contributing to shearing of the guidewire. To overcome this limitation, recently, newer 19-gauge needles and a slim echoendoscope (the EG-3270UK) have been developed.

Until now, although there have been several experimental and clinical comparative studies between 19-gauge needle and others (Trucut, 22 - and 25-gauge needles) [19-23], there has been no comparative study for various 19-gauge needles including newer ones in combination with various old and newer echoendoscopes.

Angles A and B may represent, respectively, the abilitiesto delineate and to puncture targeted lesions. Smaller angles may mean less needle maneuverability, thereby limiting the various interventions. In the present study, although initially we postulated that there was no difference between the angles $A$ and $B$ among the standard echoendoscopes equipped with a 19G needle, the standard Pentax scope (EG-3870UTK) showed optimal angulation with a variety of 19G needles compared with standard Olympus echoendoscopes. However, we should consider actual clinical situations because the echoendoscopic delineation of tar- 


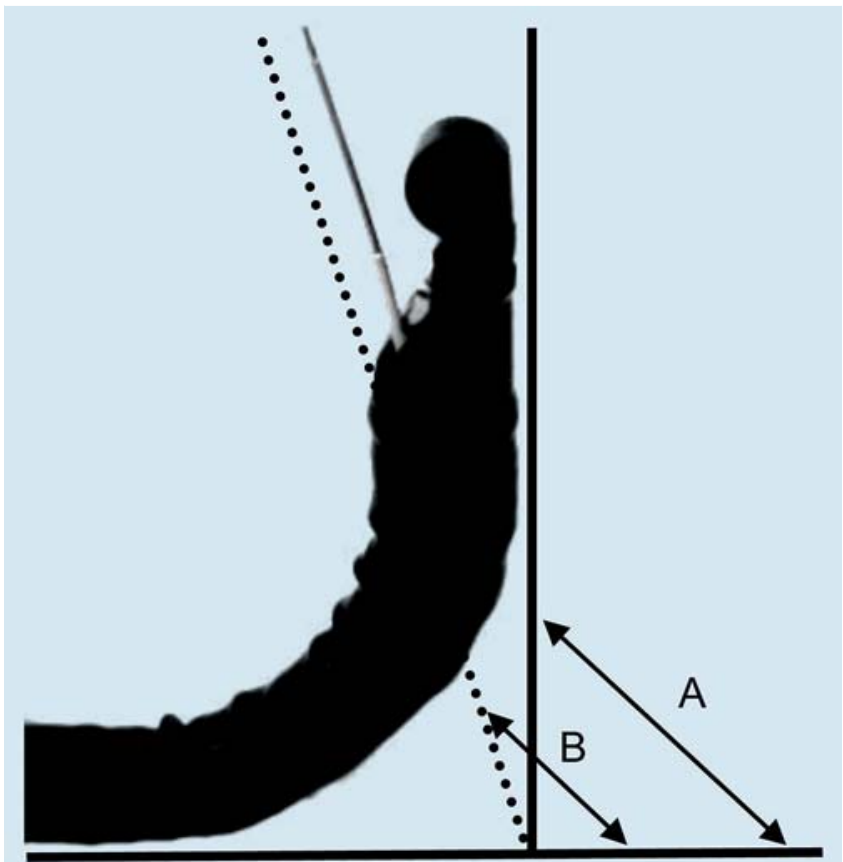

Fig.2 Measurement of angulations of distal tip of echoendoscope and needle: A, distal tip angulation; B, needle angulation.

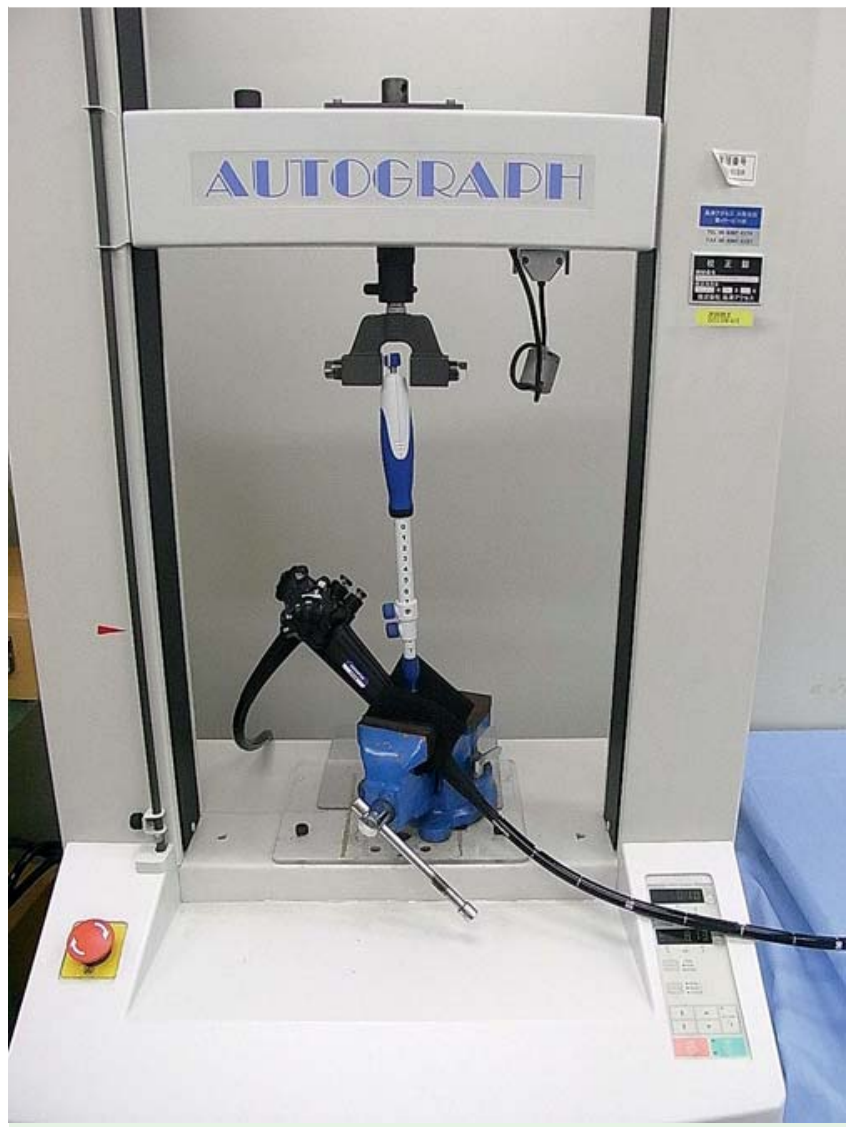

Fig.3 Autograph equipment for examination of resistance to needle insertion. geted lesions depends on both the flexibility of the scope and the direction of the ultrasound probe. The direction of the ultrasound probe differs between the Pentax and Olympus echoendoscopes, which have a side and an oblique direction, respectively, for the ultrasound beam direction. Therefore, the actual differentiation between the standard Pentax and Olympus scopes may be unclear because we did not image any real lesions by means of EUS with a 19G needle. The diagnostic slim Pentax echoendoscope (EG-3270UK) showed comparatively lower scope angulations than others. These data suggest that the standard large-diameter EUS scope has a robust wire that enables the large tip of the endoscope to be safely bent, while the diagnostic slim echoendoscope does not have a such a robust wire. That may lead to lower angulations when the 19-gauge needle is used. Since the needle angulation (angle B) is the most important parameter for various interventional EUS procedures, the Pentax slim echoendoscope in combination with a 19-gauge needle seems to be suboptimal for interventional EUS.

In terms of selection of a 19-gauge needle, from the aspect of the scope-needle angles allowed by various echoendoscopes, our data suggested that among the various 19-gauge needles, the ExpectTM flexible type needle (Boston Scientific) and/or the ProCoreTM needle (Cook Endoscopy) appear to be optimal for interventional EUS including EUS-FNA, because of the optimal angulation in various scope settings. However, the two needles are quite different in concept: theProCore ${ }^{\mathrm{TM}}$ needle is basically used for dedicated EUS-FNA procedures and the Expect ${ }^{\mathrm{TM}}$ flexible needle for both FNA and interventions. In terms of cytological/histological performance, we suggest that there may be differences between the classic 19-G needle and the ProCore ${ }^{\mathrm{TM}} 19-\mathrm{G} /$ Expect $^{\mathrm{TM}}$ 19-G flexible type needle in special settings, e.g. the trans-duodenal approach to lesion puncture. In addition, they seem to be optimal for EUS-FNA when core tissues, obtained using a 19-G needle are preferable for accurate diagnosis in special settings such as malignant lymphoma and autoimmune pancreatitis, although current 22-G and/or 25-G needles are sufficient for EUS-FNA in patients with common diseases such as pancreatic cancers.

Even when lesions can be well delineated, the puncture is not always successful because of lack of sharpness of the needle and the resistance to its advancement. Resistance to the needle is caused by various factors, e.g. torque, kinking of working channel due to scope position, and use of distal tip/elevator angulation. These limited the performance of the needle, resulting in failure of puncture during EUS-FNA. Our previous study evaluating resistance to the needle revealed that resistance to needle advancement was moderate at s resistance of $5 \mathrm{~N}$ or more and high at $8 \mathrm{~N}$ or more, and that it was impossible to advance the needle when the resistance was $11 \mathrm{~N}$ or more [19]. In the short scope position, the transgastric approach, unless the maximum up-angulation was applied in the distal tip and the elevator at the same time, needle punctures was possible using any 19-gauge needle. The results of the present study were similar to our previous data [19]. In the straight scope position, the new flexible 19-gauge needle encountered comparatively less resistance than other needles and all needles encountered lower resistance $(<8 \mathrm{~N})$. When the scope was curved, most of the 19G needles met resistance greater than $10 \mathrm{~N}$. In particular, in the case of both distaltipup and elevator-up angulation, resistances to the needle were more than $11 \mathrm{~N}$, meaning that puncture was impossible. Also surprisingly, the $R_{\text {needle }}$ for the new flexible 19-gauge needle was lower than that of others. Our results suggest that for needle 
Table 2 Angles of echoendoscope and needle using the various 19-guage EUS needles in the straight scope position.

\begin{tabular}{|c|c|c|c|c|c|c|c|c|}
\hline & \multicolumn{4}{|c|}{ Angle of echoendoscope (angle A) (degree) } & \multicolumn{4}{|c|}{ Angle of needle (angle B) (degree) } \\
\hline & Ex-19G & ExF-19G & ET-19G & PC-19G & Ex-19G & ExF-19G & ET-19G & PC-19G \\
\hline \multicolumn{9}{|l|}{ Olympus } \\
\hline GF-UCT260 & 93 & 96 & 90 & 94 & 113 & 115 & 114 & 116 \\
\hline GF-UCT240 & 77 & 90 & 88 & 92 & 96 & 115 & 108 & 115 \\
\hline GF-UCP240 & 85 & 92 & 88 & 90 & 105 & 115 & 105 & 115 \\
\hline \multicolumn{9}{|l|}{ Pentax } \\
\hline EG-3870UTK & 90 & 100 & 100 & 110 & 113 & 125 & 135 & 136 \\
\hline EG-3270UK & 55 & 70 & 72 & 80 & 95 & 95 & 104 & 104 \\
\hline
\end{tabular}

Abbreviations: ET, EchoTip; EUS, endoscopic ultrasonography; EX, Expect; ExF, Expect Flex; G, gauge; PC, ProCore.

Table 3 Angles of echoendoscope and needle using the various 19-gauge EUS needles in the curved scope position.

\begin{tabular}{|c|c|c|c|c|c|c|c|c|}
\hline & \multicolumn{4}{|c|}{ Angle of echoendoscope (angle A) (degree) } & \multicolumn{4}{|c|}{ Angle of needle (angle B) (degree) } \\
\hline & Ex-19G & ExF-19G & ET-19G & PC-19G & Ex-19G & ExF-19G & ET-19G & PC-19G \\
\hline \multicolumn{9}{|l|}{ Olympus } \\
\hline GF-UCT260 & 92 & 95 & 82 & 90 & 108 & 112 & 100 & 115 \\
\hline GF-UCT240 & 78 & 90 & 84 & 87 & 104 & 104 & 106 & 115 \\
\hline GF-UCP240 & 80 & 80 & 80 & 88 & 105 & 104 & 106 & 111 \\
\hline \multicolumn{9}{|l|}{ Pentax } \\
\hline EG-3870UTK & 85 & 95 & 88 & 92 & 105 & 124 & 125 & 130 \\
\hline EG-3270UK & 55 & 65 & 68 & 70 & 75 & 90 & 94 & 91 \\
\hline
\end{tabular}

Abbreviations: ET, EchoTip; EUS, endoscopic ultrasonography; EX, Expect; ExF, Expect Flex; G, gauge; PC, ProCore.

Table 4 The maximum resistance to advancement of needle in the straight scope position (in newtons, $\mathrm{N}$, mean \pm SD).

\begin{tabular}{|c|c|c|c|c|c|c|}
\hline Echoendoscope & $\begin{array}{l}\text { Distal tip } \\
\text { angulation }\end{array}$ & $\begin{array}{l}\text { Elevator } \\
\text { angulation }\end{array}$ & Ex-19G & ExF-19G & ET-19G & PC-19G \\
\hline \multicolumn{7}{|l|}{ Olympus } \\
\hline \multirow{4}{*}{ GF-UCT260 } & \multirow{2}{*}{$0^{\circ}$} & $0^{\circ}$ & $5.03 \pm 0.53$ & $2.01 \pm 0.21$ & $3.04 \pm 0.05$ & $3.35 \pm 0.09$ \\
\hline & & $\operatorname{Max}$ & $10.73 \pm 0.34$ & $4.10 \pm 0.20$ & $6.60 \pm 0.43$ & $7.03 \pm 0.13$ \\
\hline & \multirow{2}{*}{ Max } & $0^{\circ}$ & $7.78 \pm 0.27$ & $4.75 \pm 0.53$ & $5.04 \pm 0.14$ & $6.78 \pm 0.15$ \\
\hline & & Max & $11.55 \pm 0.52$ & $5.13 \pm 0.13$ & $6.12 \pm 0.10$ & $7.59 \pm 0.22$ \\
\hline \multirow{4}{*}{ GF-UCT240 } & \multirow{2}{*}{$0^{\circ}$} & $0^{\circ}$ & $4.68 \pm 0.20$ & $2.75 \pm 0.10$ & $4.48 \pm 0.16$ & $2.81 \pm 0.17$ \\
\hline & & Max & $8.60 \pm 0.43$ & $4.34 \pm 0.19$ & $8.28 \pm 0.40$ & $6.23 \pm 0.32$ \\
\hline & \multirow{2}{*}{ Max } & $0^{\circ}$ & $7.00 \pm 0.21$ & $4.98 \pm 0.45$ & $7.48 \pm 0.21$ & $5.05 \pm 0.10$ \\
\hline & & Max & $9.47 \pm 0.12$ & $5.61 \pm 0.36$ & $9.09 \pm 0.19$ & $6.93 \pm 0.08$ \\
\hline \multirow{4}{*}{ GF-UCP240 } & \multirow{2}{*}{$0^{\circ}$} & $0^{\circ}$ & $7.82 \pm 0.25$ & $1.61 \pm 0.09$ & $4.38 \pm 0.18$ & $3.14 \pm 0.43$ \\
\hline & & Max & $9.30 \pm 0.37$ & $3.78 \pm 0.21$ & $6.93 \pm 0.03$ & $5.58 \pm 0.08$ \\
\hline & \multirow{2}{*}{ Max } & $0^{\circ}$ & $13.63 \pm 0.40$ & $3.77 \pm 0.08$ & $7.58 \pm 0.52$ & $5.40 \pm 0.09$ \\
\hline & & Max & $14.58 \pm 0.20$ & $5.60 \pm 0.87$ & $8.58 \pm 0.10$ & $6.87 \pm 0.10$ \\
\hline \multicolumn{7}{|l|}{ Pentax } \\
\hline \multirow{4}{*}{ EG-3870UTK } & \multirow{2}{*}{$0^{\circ}$} & $0^{\circ}$ & $4.23 \pm 0.25$ & $1.57 \pm 0.08$ & $3.12 \pm 0.06$ & $2.83 \pm 0.16$ \\
\hline & & $\operatorname{Max}$ & $6.02 \pm 0.39$ & $2.98 \pm 0.03$ & $6.05 \pm 0.05$ & $4.77 \pm 0.08$ \\
\hline & \multirow{2}{*}{ Max } & $0^{\circ}$ & $7.98 \pm 0.30$ & $3.53 \pm 0.33$ & $6.83 \pm 0.13$ & $4.28 \pm 0.53$ \\
\hline & & Max & $9.30 \pm 0.99$ & $4.05 \pm 0.23$ & $8.55 \pm 0.23$ & $7.03 \pm 0.10$ \\
\hline \multirow{4}{*}{ EG-3270UK } & \multirow{2}{*}{$0^{\circ}$} & $0^{\circ}$ & $3.63 \pm 0.21$ & $1.57 \pm 0.03$ & $4.10 \pm 0.15$ & $2.82 \pm 0.13$ \\
\hline & & Max & $5.00 \pm 0.17$ & $7.30 \pm 0.64$ & $10.90 \pm 0.20$ & $8.42 \pm 0.23$ \\
\hline & \multirow{2}{*}{$\operatorname{Max}$} & $0^{\circ}$ & $7.95 \pm 0.30$ & $3.68 \pm 0.08$ & $7.33 \pm 0.08$ & $5.23 \pm 0.13$ \\
\hline & & Max & $8.88 \pm 0.33$ & $7.42 \pm 1.01$ & $10.93 \pm 0.17$ & $9.32 \pm 0.13$ \\
\hline
\end{tabular}

Abbreviations: ET, EchoTip; Ex, Expect; ExF, Expect Flex; Max, maximum angulation; PC, ProCore. 
Table 5 The maximum resistance to advancement of needle in the curved scope position (in newtons $\mathrm{N}$, mean \pm SD)

\begin{tabular}{|c|c|c|c|c|c|c|}
\hline Echoendoscope & $\begin{array}{l}\text { Distal tip } \\
\text { angulation }\end{array}$ & $\begin{array}{l}\text { Elevator } \\
\text { angulation }\end{array}$ & Ex-19G & ExF-19G & ET-19G & PC-19G \\
\hline \multicolumn{7}{|l|}{ Olympus } \\
\hline \multirow{4}{*}{ GF-UCT260 } & \multirow{2}{*}{$0^{\circ}$} & $0^{\circ}$ & $10.29 \pm 0.32$ & $2.51 \pm 0.17$ & $5.23 \pm 0.15$ & $3.83 \pm 0.22$ \\
\hline & & $\operatorname{Max}$ & $13.52 \pm 0.49$ & $7.84 \pm 0.52$ & $12.78 \pm 0.51$ & $11.75 \pm 0.14$ \\
\hline & \multirow{2}{*}{ Max } & $0^{\circ}$ & $10.61 \pm 0.74$ & $5.29 \pm 0.09$ & $7.39 \pm 0.15$ & $7.29 \pm 0.23$ \\
\hline & & $\operatorname{Max}$ & $14.53 \pm 0.77$ & $9.16 \pm 0.49$ & $12.80 \pm 0.34$ & $11.91 \pm 0.63$ \\
\hline \multirow{4}{*}{ GF-UCT240 } & \multirow{2}{*}{$0^{\circ}$} & $0^{\circ}$ & $10.13 \pm 0.62$ & $3.05 \pm 0.17$ & $6.91 \pm 0.54$ & $4.61 \pm 0.34$ \\
\hline & & Max & $12.37 \pm 0.38$ & $10.55 \pm 0.34$ & $16.35 \pm 0.76$ & $12.08 \pm 0.97$ \\
\hline & \multirow{2}{*}{ Max } & $0^{\circ}$ & $13.80 \pm 0.22$ & $7.47 \pm 0.10$ & $10.65 \pm 0.66$ & $8.33 \pm 0.34$ \\
\hline & & $\operatorname{Max}$ & $15.23 \pm 0.33$ & $11.42 \pm 0.08$ & $17.35 \pm 0.44$ & $14.43 \pm 0.86$ \\
\hline \multirow{4}{*}{ GF-UCP240 } & \multirow{2}{*}{$0^{\circ}$} & $0^{\circ}$ & $8.87 \pm 0.32$ & $3.39 \pm 0.20$ & $7.66 \pm 0.23$ & $4.53 \pm 0.18$ \\
\hline & & Max & $12.05 \pm 0.40$ & $9.25 \pm 0.15$ & $14.50 \pm 0.83$ & $10.10 \pm 0.15$ \\
\hline & \multirow{2}{*}{ Max } & $0^{\circ}$ & $12.45 \pm 0.26$ & $6.23 \pm 0.06$ & $10.22 \pm 0.20$ & $7.60 \pm 0.21$ \\
\hline & & Max & $14.70 \pm 0.25$ & $12.15 \pm 0.72$ & $15.11 \pm 0.71$ & $11.67 \pm 0.24$ \\
\hline \multicolumn{7}{|l|}{ Pentax } \\
\hline \multirow{4}{*}{ EG-3870UTK } & \multirow{2}{*}{$0^{\circ}$} & $0^{\circ}$ & $10.77 \pm 0.12$ & $2.03 \pm 0.08$ & $5.64 \pm 0.07$ & $3.15 \pm 0.10$ \\
\hline & & $\operatorname{Max}$ & $12.62 \pm 0.15$ & $5.43 \pm 0.10$ & $12.30 \pm 0.30$ & $9.33 \pm 0.53$ \\
\hline & \multirow{2}{*}{ Max } & $0^{\circ}$ & $14.95 \pm 0.48$ & $4.55 \pm 0.09$ & $9.18 \pm 0.08$ & $7.10 \pm 0.05$ \\
\hline & & $\operatorname{Max}$ & $16.00 \pm 0.35$ & $5.88 \pm 0.26$ & $11.35 \pm 0.20$ & $9.95 \pm 0.36$ \\
\hline \multirow{4}{*}{ EG-3270UK } & \multirow{2}{*}{$0^{\circ}$} & $0^{\circ}$ & $7.72 \pm 0.53$ & $2.47 \pm 0.08$ & $4.47 \pm 0.23$ & $3.33 \pm 0.10$ \\
\hline & & Max & $11.27 \pm 0.31$ & $10.11 \pm 0.55$ & $15.80 \pm 0.22$ & $14.78 \pm 0.28$ \\
\hline & \multirow{2}{*}{ Max } & $0^{\circ}$ & $11.65 \pm 0.54$ & $6.65 \pm 0.17$ & $9.70 \pm 0.23$ & $8.05 \pm 0.22$ \\
\hline & & $\operatorname{Max}$ & $13.00 \pm 0.18$ & $12.08 \pm 0.40$ & $17.77 \pm 0.63$ & $13.12 \pm 0.51$ \\
\hline
\end{tabular}

Abbreviations: ET, EchoTip; EX, Expect; ExF, Expect Flex; Max, maximum angulation; PC, ProCore.

puncture in difficult situations, such as trans-duodenal approach, the flexible 19G needle may be most appropriate. However, even when the flexible 19-gauge needle is used, the tight bending of distal tip-up and elevator-up angulation hinders advancement of the needle and at times, puncture by the needle is impossible, in particular when diagnostic thinner echoendoscopes (GF-UCP240 and EG-3270UK) are used. We speculate that in such thinner scopes, the needle compliance decreases because of kinking of the smaller working channel by distal tip angulation, or because of strong friction when the the needle is pinned by the elevator mechanism.

The up-to-date findings of our study may be important because they are an important step in knowing which 19-gauge needles and endoscopes are better for EUS-related procedures. Our results suggest that both the Expect ${ }^{\mathrm{TM}}$ flexible type needle and the ProCore ${ }^{\mathrm{TM}}$ needle seem to be optimal for EUS-FNA used with either therapeutic Olympus (GF-UCT260 and GF-UCT240) or Pentax scopes (EG-3870UTK). However, for interventional EUS that requires the use of a guidewire, the Expect ${ }^{\mathrm{TM}}$ flexible needle appears to be optimal, in particular for the trans-duodenal approach.

The current 19-gauge needles, however, are still not the best for EUS-related procedures. For instance, the $19 \mathrm{G}$ ProCore ${ }^{\mathrm{TM}}$ needle and the Expect ${ }^{\mathrm{TM}} 19 \mathrm{G}$ flexible needle are still not as flexible compared to current 22 - and 25-gauge needles. Thus, we still need of better technology to overcome this limitation.

There are several limitations in this study. Since various factors, such as echoendoscope position, the patient's pathology, anatomical differences, and torque of the scope cannot be reproduced in an experimental model, the conclusions may not hold true for all clinical scenarios.

In conclusion, our experimental data suggests that both the Expect $^{\mathrm{TM}}$ flexible type needle and the ProCore ${ }^{\mathrm{TM}}$ needle seems to be most suitable for EUS-FNA procedures. The Expect ${ }^{\mathrm{TM}}$ flexible type needle appears to be particularly suitable for the trans-duodenal approach and for interventional EUS procedures.

\section{Competing interests: None.}

\section{Acknowledgement}

The authors are indebted to Professor J. Patrick Barron of the International Medical Communications Center of Tokyo Medical University for his review of this manuscript.

\section{References}

1 Iwashita T, Yasuda I, Doi S et al. Use of samples from endoscopic ultrasound-guided 19-gauge fine-needle aspiration in diagnosis of autoimmune pancreatitis. Clin Gastroenterol Hepatol 2012; 10: 316-322

2 Varadarajulu S, Bang JY, Hebert-Magee S. Assessment of the technical performance of the flexible 19-gauge EUS-FNA needle. Gastrointest Endosc 2012; 76: 336-343

3 Varadarajulu S, Lopes TL, Wilcox CM et al. EUS versus surgical cyst-gastrostomy for management of pancreatic pseudocysts. Gastrointest Endosc 2008; 68: 649-655

4 Itoi T, Binmoeller KF, Shah J et al. Clinical evaluation of a novel lumenapposing metal stent for endosonography-guided pancreatic pseudocyst and gallbladder drainage (with video). Gastrointest Endosc 2012; 75: $870-876$

5 Park DH, Lee SS, Moon SH et al. Endoscopic ultrasound-guided versus conventional transmural drainage for pancreatic pseudocysts: a prospective randomized trial. Endoscopy 2009; 41: $842-848$

6 Seewald S, Ang TL, Richter $H$ et al. Long-term results after endoscopic drainage and necrosectomy of symptomatic pancreatic fluid collections. Dig Endosc 2012; 24: 36-41

7 Giovannini M, Dotti M, Bories E et al. Hepaticogastrostomy by echoendoscopy as a palliative treatment in a patient with metastatic biliary obstruction. Endoscopy 2003; 35: 1076-1078

8 Horaguchi J, Fujita N, Noda Y et al. Endosonography-guided biliary drainage in cases with difficult transpapillary endoscopic biliary drainage. Dig Endosc 2009; 21: 239 - 244 
9 Itoi T, Isayama $\mathrm{H}$, Sofuni $A$ et al. Stent selection and tips on placement technique of EUS-guided biliary drainage: transduodenal and transgastric stenting. J Hepatobiliary Pancreat Sci 2011; 18: 664-672

10 Artifon EL, Pinhata OtochJ et al. Endoscopy Ultra- Sonography -guided biliary drainage in the surgical -endoscopy era. Rev Gastroenterol Peru 2001; 31: 365-375

11 Park $\mathrm{DH}, \mathrm{Koo} \mathrm{JE}, \mathrm{Oh} \mathrm{J}$ et al. EUS-guided biliary drainage with one-step placement of a fully covered metal stent for malignant biliary obstruction: A prospective feasibility study. Am J Gastroenterol 2009; 104: $2168-2174$

12 Hara K, Yamao K, Niwa Yet al. Prospective clinical study of EUS-guided choledochoduodenostomy for malignant lower biliary tract obstruction. Am J Gastroenterol 2011; 106: 1239-1245

13 Kahaleh $M$. Therapeutic and advanced endoscopic retrograde cholangiopancreatography. Gastrointest Endosc Clin N Am. 2012 Jul22: xv

14 Baron TH, Topazian MD. Endoscopic transduodenal drainage of the gallbladder: implications for endoluminal treatment of gallbladder disease. Gastrointest Endosc 2007; 65: 735 -737

15 Eum J, Park doHyun, Ryu CH et al. EUS-guided biliary drainage with a fully covered metal stent as a novel route for natural orifice transluminal endoscopic biliary interventions: a pilot study (with videos). Gastrointest Endosc 2011; 72: 1279-1284

16 Itoi T, Itokawa F, Kurihara T. Endoscopic ultrasonography-guided gallbladder drainage: actual technical presentations and review of the literature (with videos). J Hepatobiliary Pancreat Sci 2011; 18: 282 - 286
17 Giovannini M. Endoscopic ultrasonography-guided pancreatic drainage. Gastrointest Endosc Clin N Am 2012; 22: 221 - 230

18 Itoi T, Kikuyama M, Ishii $K$ et al. EUS-guided rendezvous with singleballoon enteroscopy for treatment of stenotic pancreaticojejunal anastomosis in post-Whipple patients (with video). Gastrointest Endosc 2011; 73: $398-401$

19 Itoi $T$, Itokawa $F$, Kurihara $T$ et al. Experimental endoscopy: objective evaluation of EUS needles. Gastrointest Endosc 2009; 69: 509-516

20 Varadarajulu S, Fraig M, Schmulewitz $N$ et al. Comparison of EUS-guided 19-gauge trucut needle biopsy with EUS-guided fine-needle aspiration. Endoscopy 2004; 36: 397-401

21 Itoi T, Itokawa H, Sofuni A et al. Puncture of solid pancreatic tumors guided by endoscopic ultrasonography: A pilot study series comparing trucut and 19-gauge and 22-gauge aspiration needle. Endoscopy 2005; 37: 362 - 366

22 Song TJ, Kin J, Lee SS et al. The prospective tandomized, controlled trial of endoscopic ultrasound-guided fine-needle aspiration using $22 \mathrm{G}$ and 19G aspiration needles for solid pancreatic or peripancreatic masses. Am J Gastroenterol 2010; 105: 1739-1745

23 Sakamoto H, Kitano M, Komaki $T$ et al. Prospective comparative study of the EUS guided 25-gauge FNA needle with the 19-gauge Trucut needle and 22-gauge FNA needle in patients with solid pancreatic masses. J Gastroenterol Hepatol 2009; 24: 384-390 